\title{
No Sterile Neutrinos from Eight Years of IceCube
}

\author{
An analysis of more than 300,000 muon neutrino detections provides no \\ evidence of sterile neutrinos-a finding at odds with other experiments.
}

\author{
By Erika K. Carlson
}

$\square$ xperiments that study neutrino oscillations have previously suggested the existence of a "sterile" neutrino that doesn't interact via the weak nuclear force-unlike the three familiar varieties. A 2016 survey by the IceCube Neutrino Observatory in Antarctica failed to reproduce these observations, however (see Viewpoint: Hunting the Sterile Neutrino). Now, the IceCube Collaboration has searched for signs of this elusive particle within a dataset 15 times larger than that analyzed in the 2016 study. Still finding no evidence for sterile neutrinos, the team's result further exacerbates the tension between earlier experiments and IceCube's seemingly contradictory results $[1,2]$.

IceCube detects neutrinos that originate mostly in collisions between cosmic rays and the Earth's atmosphere. As well as measuring the neutrinos' energies, the experiment can determine their trajectories, which tell researchers how much of the Earth the particles passed through before reaching the detectors. These two factors determine how many muon

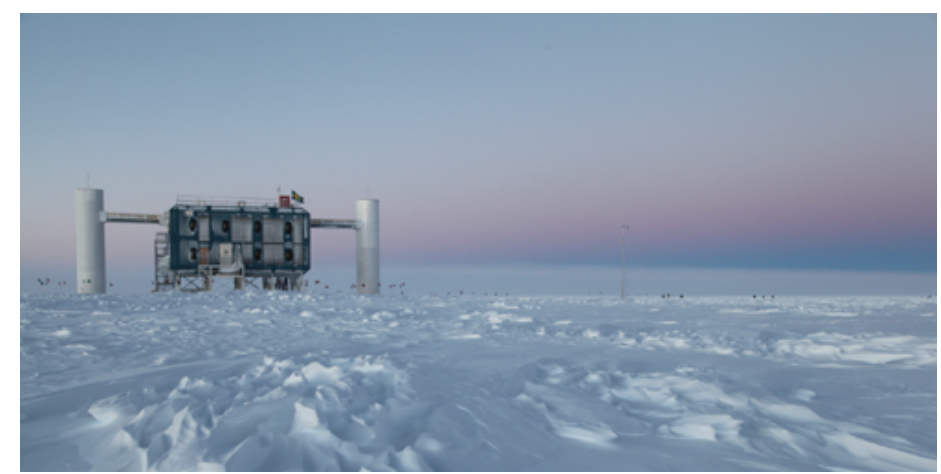

Credit: Sven Lindstrom/IceCube/NSF neutrinos the observatory should detect. But theory predicts that the presence of sterile neutrinos would make more of the muon neutrinos oscillate into other neutrino flavors, skewing the numbers. Therefore, if the detectors observe fewer muon neutrinos than predicted, it could indicate that sterile neutrinos exist. After analyzing more than 300,000 muon neutrino detections logged over eight years, the researchers find no significant deviation in the number of muon neutrino events from theoretical predictions and, thus, no evidence for sterile neutrinos.

This continuing lack of evidence widens the discrepancy between IceCube's results and those from the Liquid Scintillator Neutrino Detector (LSND) and MiniBooNE experiments, both of which have found hints of sterile neutrinos (see Viewpoint: The Plot Thickens for a Fourth Neutrino). For the IceCube Collaboration, next steps include expanding their analysis to detections of electron and tau neutrinos, an excess of which may also indicate the involvement of sterile neutrinos.

Erika K. Carlson is a Corresponding Editor for Physics based in New York City.

\section{REFERENCES}

1. M. Aartsen et al., "eV-scale sterile neutrino search using eight years of atmospheric muon neutrino data from the IceCube Neutrino Observatory," Phys. Rev. Lett, 125, 141801 (2020).

2. M. Aartsen et al., "Searching for eV-scale sterile neutrinos with eight years of atmospheric neutrinos at the IceCube Neutrino Telescope," Phys. Rev. D 102, 052009 (2020). 